\title{
Hybrid Monte Carlo simulation on the graphene hexagonal lattice
}

\author{
Richard Brower* \\ ECE and Physics Departments and Center for Computational Science \\ Boston University, Boston, MA 02215 \\ E-mail: brower@bu.edu
}

\section{Claudio Rebbi*}

Physics Department and Center for Computational Science

Boston University, Boston, MA 02215

E-mail: rebbiabu . edu

\section{David Schaich}

Physics Department

University of Colorado, Boulder, CO 80309

E-mail: David.Schaich@Colorado.EDU

\begin{abstract}
One of the many remarkable properties of graphene is that in the low energy limit the dynamics of its electrons can be effectively described by the massless Dirac equation. This has prompted investigations of graphene based on the lattice simulation of a system of 2-dimensional fermions on a square staggered lattice. We demonstrate here how to construct the path integral for graphene working directly on the graphene hexagonal lattice. For the nearest neighbor tight binding model interacting with a long range Coulomb interaction between the electrons, this leads to the hybrid Monte Carlo algorithm with no sign problem. The only approximation is the discretization of the Euclidean time. So as we extrapolate to the time continuum limit, the exact tight binding solution maybe found numerically to arbitrary precession on a finite hexagonal lattice. The potential for this approach is tested on a single hexagonal cell.
\end{abstract}

The XXIX International Symposium on Lattice Field Theory - Lattice 2011 July 10-16, 2011 Squaw Valley, Lake Tahoe, California

\footnotetext{
* Speaker
} 


\section{Contents}

1. Introduction 2

2. The graphene lattice 3

3. The dynamics 4

4. Path integral formulation 5

$\begin{array}{llr}\text { 5. Numerical tests } & 8\end{array}$

$\begin{array}{ll}\text { 6. Discussion } & 10\end{array}$

$\begin{array}{ll}\text { 7. Conclusion } & 13\end{array}$

\section{Introduction}

During the last few years graphene, a single layer of carbon atoms forming a hexagonal lattice, has burst on the scientific scene as a remarkable material with intriguing theoretical properties and potentially astounding technological applications [1]. Its electronic structure is well approximated by the tight binding Hamiltonian $H$ which describes the quantum mechanical motion of the electrons, one per atom at half-filling, that are not part of the cloud of electrons responsible for the covalent binding of the carbon atoms. The simplest model Hamiltonian, $H=H_{2}+H_{C}$, consists of two terms, a quadratic Hamiltonian $\mathrm{H}_{2}$ which describes the hopping of the electrons between neighboring atoms, and a Hamiltonian $H_{C}$ which describes the Coulomb repulsion of the electrons. In the the limit, where the strength of the hopping term is taken to be much larger than strength of the Coulomb interaction, the quadratic Hamiltonian $\mathrm{H}_{2}$ gives origin to a dispersion formula which for low momenta is analogous to the dispersion formula for relativistic fermions in two dimensions [2]. This has prompted some researchers to adapt to graphene lattice gauge theory techniques which have been profitably used for the study of Quantum Chromodynamics and other particle systems [3]. In the work of [4, 5] one approximates the tight-binding Hamiltonian of graphene with a Dirac Hamiltonian, incorporates the Coulomb interaction through the introduction of a suitable electromagnetic field, and finally discretized the resulting continuum quantum field theory on a hypercubic space-time lattice. The hybrid Monte Carlo method [6], widely used in lattice gauge theory to simulate fermions interacting with quantum gauge fields, can then be used to investigate the graphene system.

The approach outlined above has led to very interesting and valuable results [4, 5], yet it has several limitations. First the use of staggered fermion on a cubic lattice looses the direct connection to special symmetries of hexagonal lattice and introduces symmetry breaking artifacts, know in 
lattice QCD as taste splitting [7]. Second a 4-d lattice is required to simulate the coulomb potential which is not only computational expensive but distorted at the scale of the physical spatial lattice spacing. One would think that, since the starting point is a system already defined on a lattice, it should be possible to apply the hybrid Monte Carlo technique directly to the graphene lattice. The clear advantage of working on the hexagonal graphene lattice is the direct connection to the experimentally determined physical lattice constants of the tight-binding model, which represents an accurate description of the experimental system. Here we wish to illustrate how this can be done.

\section{The graphene lattice}

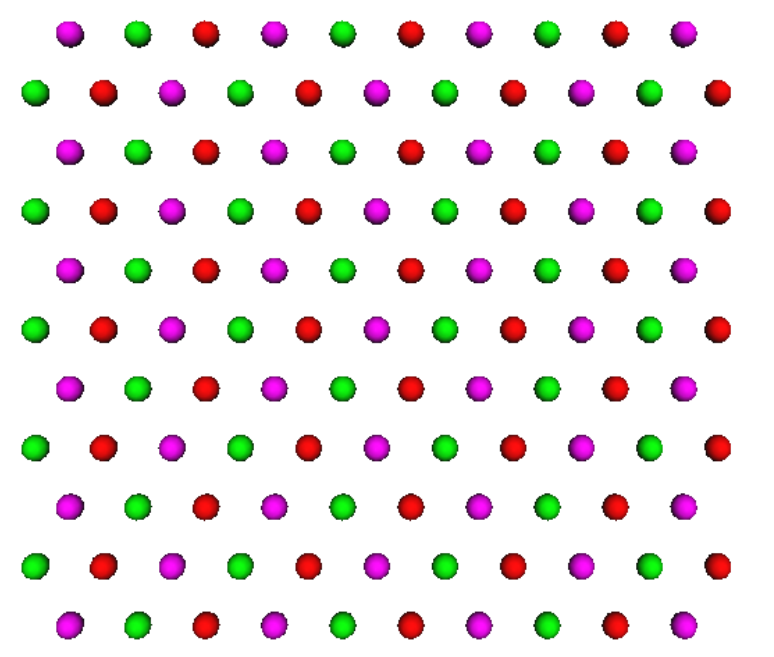

Figure 1: The underlying triangular lattice.

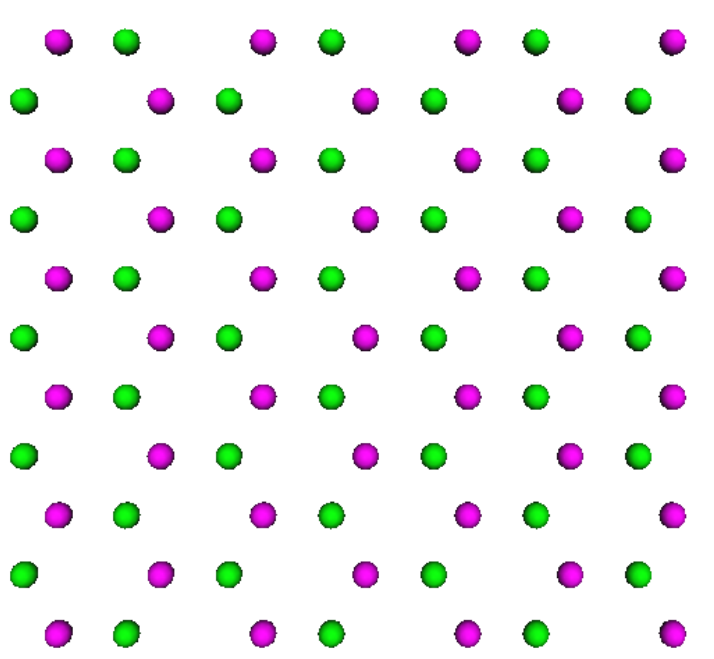

Figure 2: The hexagonal graphene lattice.

It is useful to review the geometrical properties of graphene. Graphene is a system of interacting electrons located at the vertices of a hexagonal lattice. It is convenient to think of the graphene lattice as consisting of two triangular sublattices, which we denote by $A$ and $B$, which together with the centers of the hexagons (sublattice $C$ ) form a finer, underlying triangular lattice. In Figures 1 and 2, we illustrate the full underlying triangular lattice and the hexagonal lattice which one obtains by eliminating the sites of one of the three sublattices. $A$ and $B$ are Bravais lattices so given any two primitive vectors $\vec{a}_{1}, \vec{a}_{2}$ their sites ( at $\vec{r}_{A}=x_{1} \vec{a}_{1}+x_{2} \vec{a}_{2}$ and $\vec{r}_{B}=x_{1}^{\prime} \vec{a}_{1}+x_{2}^{\prime} \vec{a}_{2}$ respectively) are conveniently enumerated by two integers $\left(x_{1}, x_{2}\right)$.

One may impose periodic boundary conditions (PBC) by identifying sites that differ by a translation by $L_{i}$ along the primitive vectors: $x_{i} \simeq x_{i}+L_{i}$. In Figure 3, we show an example of PBC for $L_{1}=L_{2}=4$ with green and purple color for the independent lattice sites on each sublattice. In this representation, each sublattice A and B is mapped into a rectangle with periodic boundary condition very easily coded by two dimensional arrays. The pattern of these sites does not look reminiscent of the hexagonal structure of graphene. However, it is equivalent. If one rearranges the sites by replacing some of the vertices with their periodic images and one adds to these vertices a few of their periodic images one obtains a pattern of sites which clearly shows the hexagonal structure of graphene with 3 fold rotational symmetry as illustrated in Figure 4. Of course, one could 


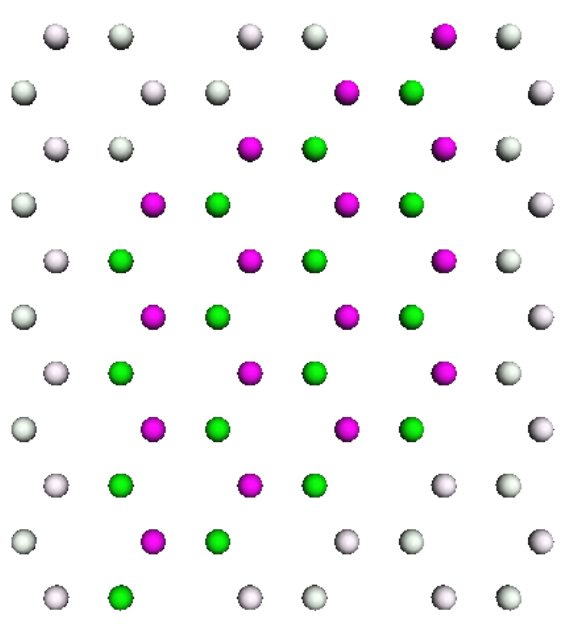

Figure 3: Periodic lattice with $2 L_{1} L_{2}$ sites viewed as parallelogram subdomain in the plane illustrated for period $L_{i}=4$ along two primitive vectors: $\hat{a}_{1} \sim$ $\sqrt{3} \hat{x} / 2+\hat{y} / 2, \hat{a}_{2} \sim \hat{y}$, defined relative to the Cartesian unit vectors, $(\hat{x}, \hat{y})$.

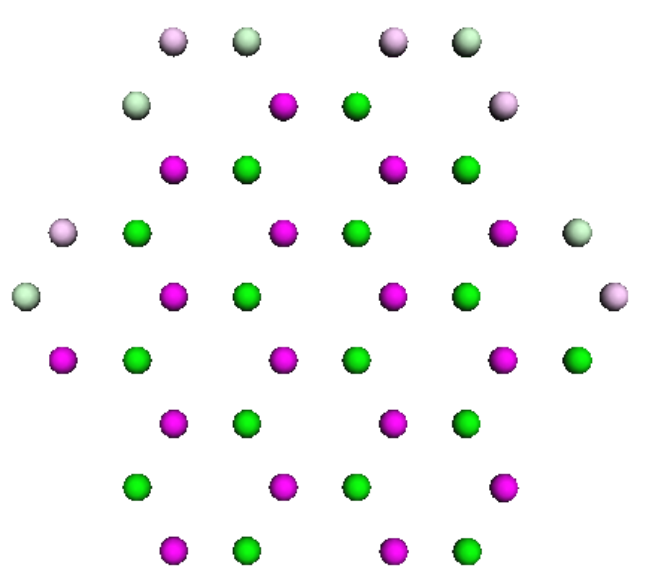

Figure 4: The sublattice with period $L=4$ from Figure 3 is mapped into periodic hexagonal domain by moving four vertices to equivalent sites combined with ten image or ghost sites shown in lighter color to complete the pattern.

also introduce different periods $L_{1}, L_{2}$ along two directions of the fundamental cell and consider other types of boundary conditions. For example, by taking $L_{2} \gg L_{1}$ with PBC along the shortest dimension and open boundary conditions direction along the other one can simulate a nanotube.

\section{The dynamics}

We introduce fermionic annihilation and creation operators $a_{x, s}, a_{x, s}^{\dagger}$ for the electrons on the two sublattices, where $x$ is a site index for both the A and B sublattices and $s= \pm 1$ labels the spin of the electrons. The Hamiltonian $H=H_{2}+H_{C}$ consists of two terms. The quadratic kinetic term is

$$
H_{2}=\sum_{\langle x, y\rangle, s}-s \kappa\left(a_{x, s}^{\dagger} a_{y, s}+\text { h.c. }\right)
$$

where the sum runs over all pairs $\langle x, y\rangle$ of nearest neighbor sites (coupling the $A$ and $B$ sublattices) and the two values of the spin. The Coulomb interaction term is

$$
H_{C}=\sum_{x, y} e^{2} V_{x, y} q_{x} q_{y}=\frac{1}{2} \sum_{x \neq y} q_{x} \frac{e^{2}}{\sqrt{\left(\vec{r}_{x}-\vec{r}_{y}\right)}} q_{y}+\sum_{x} q_{x} \frac{e^{2}}{r_{0}} q_{x}
$$

where $q_{x}=a_{x, 1}^{\dagger} a_{x, 1}+a_{x,-1}^{\dagger} a_{x,-1}-1$ is a local charge operator and $V$ is the interaction potential with physical co-ordinates $\vec{r}_{x}, \vec{r}_{y}$ for each Carbon atom. Note that we have introduced a -1 in the charge operator $q_{x}$ to account for the background charge of the carbon ion: this ensures that the system is neutral at half filling, and it will play an important role for our functional integral formulation. Insofar as the matrix $V$ in Eq. 3.2 is concerned, it could be the actual 3d Coulomb potential, but could be any other interaction potential. The only constraint is that the matrix $V_{x, y}$ must be positive definite. 
The phenomenological constants are a nearest neighbor lattice spacing of $a \simeq 1.42, \kappa \simeq$ $2.8 \mathrm{Mev}$ and $e^{2}$ is effective parameter for $e^{2} / \hbar v \simeq 300 \times e^{2} / \hbar c$ so that unlike free electrons effective charge is very strong and the electrodynamics essential static, free of magnetic effects. The single site Coulomb [8] radius, $r_{0} \simeq 0.5 a$, is essential for stability of the vacuum configuration. We also note that in Eq. 3.1 we have neglected the smaller next-to-nearest neighbor hopping within each sublattice, estimated to be $\kappa^{\prime} \simeq 0.03 \kappa$. This would introduce a (probably manageable) complex phase in the path integral.

Finally, we observe that the Hamiltonian of Eqs. 3.1-3.2 commutes with the angular momentum generators,

$$
\begin{aligned}
J_{ \pm} & =a_{x, s}^{\dagger} \sigma_{ \pm}^{s s^{\prime}} a_{x, s^{\prime}} \\
J_{3} & =a_{x, s}^{\dagger} \sigma_{3}^{s s^{\prime}} a_{x, s^{\prime}} / 2
\end{aligned}
$$

which act on the spin of the electron and play the role in the tight-binding model of an internal or "isospin" symmetry. Additional symmetries of the system are the overall momentum conservation, parity under reflection with respect to the crystal axes and under interchange of the $A$ and $B$ sublattices, and the separate conservations of the number of electrons with spin up and spin down.

In order to explore the properties of the system one would like to calculate expectation values

$$
\left\langle\mathscr{O}_{1}\left(t_{1}\right) \mathscr{O}_{2}\left(t_{2}\right) \ldots\right\rangle=Z^{-1} \operatorname{Tr} T\left[\mathscr{O}_{1}\left(t_{1}\right) \mathscr{O}_{2}\left(t_{2}\right) e^{-\beta H}\right],
$$

where $\mathscr{O}$ stands for a generic observable, $\beta$ can be interpreted as an extent in Euclidean time, $T[\ldots]$ stands for time ordering of the operators inside the square bracket with respect to the Euclidean evolution implemented by $\exp (-\beta H)$, and $Z=\operatorname{Tr} \exp (-\beta H)$ is the partition function.

\section{Path integral formulation}

Our goal is to provide an equivalent path integral formulation of Eq. 3.4 conducive to calculation by numerical simulation, following a rather standard procedure to convert from the Hamiltonian into a Lagrangian. We will first express the expectation values and the partition function in terms of an integral over anticommuting fermionic fields, i.e. elements of a Grassmann algebra. (See for example [9].) It is important that the Hamiltonian be normal ordered. This is true of $H_{2}$ and of the non-local terms in $H_{C}$. However the normal ordered : $H_{C}$ : differs from $H_{C}$ by a diagonal quadratic term which can be added to $H_{2}$. With this in mind one can proceed to the path integral formulation, which gives origin to an integral over the Grassmann variables of an exponential containing a quadratic form in the fermionic fields, from $H_{2}$ and the normal ordering of $H_{C}$, as well as a quartic expression from : $H_{C}:$. The quartic expression can be reduced to a quadratic form by a Hubbard-Stratonovich transformation [10], through the introduction of a suitable auxiliary bosonic field (in our case a real field), and now the Gaussian integral over the fermionic variables can be explicitly performed, leaving an integral over the bosonic field only [11]. The problem, however, is to obtain an integral that can be interpreted as an integration over a well defined probabilistic measure, which can be approximated by stochastic simulation techniques. In the following we show how this can be accomplished by taking advantage of the symmetries of the system 
We start by rewriting the expression for the charge as

$$
q_{x}=a_{x, 1}^{\dagger} a_{x, 1}-a_{x,-1} a_{x,-1}^{\dagger} .
$$

We now introduce hole creation and annihilation operators for the electrons with spin -1 :

$$
b_{x}^{\dagger}=a_{x,-1}, \quad b_{x}=a_{x,-1}^{\dagger}
$$

so that the charge becomes

$$
q_{x}=a_{x}^{\dagger} a_{x}-b_{x}^{\dagger} b_{x} .
$$

Note that we dropped the spin indices since from now on $a, a^{\dagger}$ and $b, b^{\dagger}$ will always refer to spin 1 and -1 , respectively. Finally we change the sign of the $b, b^{\dagger}$ operators on one of the sublattices. The crucial constraint is that all redefinitions of the operators respect the anticommutator algebra. Because $\mathrm{H}_{2}$ only couples sites on the two different sublattices, it takes the form

$$
H_{2}=\sum_{\langle x, y\rangle}-\kappa\left(a_{x}^{\dagger} a_{y}+b_{x}^{\dagger} b_{y}+\text { h.c. }\right)
$$

We introduce fermionic coherent states

$$
\left\langle\psi^{*}, \eta^{*}\left|=\left\langle 0\left|e^{-\sum_{x}\left(a_{x} \psi_{x}+b_{x} \eta_{x}\right)} \quad\right| \psi, \eta\right\rangle=e^{-\sum_{x}\left(\psi_{x} a_{x}^{\dagger}+\eta_{x} b_{x}^{\dagger}\right)}\right| 0\right\rangle,
$$

where $\psi_{x}, \psi_{x}^{*}, \eta_{x}, \eta_{x}^{*}$ are anticommuting fermionic variables (elements of a Grassmann algebra). The path integral formulation is obtained by factoring

$$
e^{-\beta H}=e^{-H \delta} e^{-H \delta} \ldots e^{-H \delta} \quad\left(N_{t} \text { terms }\right)
$$

with $\delta=\beta / N_{t}$, and then inserting repeatedly among the factors the resolution of the identity expressed in terms of an integral over the fermionic variables. The trace in Eq. 3.4 must also be expressed in terms of a similar integral (see e.g. [9] for details). This leads to integrals over fermionic fields $\psi_{x, t}, \psi_{x, t}^{*}, \eta_{x, t}, \eta_{x, t}^{*}$ (the index $t=0, \cdots, N_{t}-1$ appears because of the multiple resolutions of the identity and can be thought of as an index labeling Euclidean time), which contain in the integrand expressions of the type

$$
\left\langle\psi_{x, t}^{*}, \eta_{x, t}^{*}\left|e^{-H \delta}\right| \psi_{x, t}, \eta_{x, t}\right\rangle
$$

The last ingredient is the identity

$$
\left\langle\psi_{x, t}^{*}, \eta_{x, t}^{*}\left|F\left(a_{x}^{\dagger}, b_{x}^{\dagger}, a_{x}, b_{x}\right)\right| \psi_{x, t}, \eta_{x, t}\right\rangle=F\left(\psi_{x, t}^{*}, \eta_{x, t}^{*}, \psi_{x, t}, \eta_{x, t}\right) e^{\sum_{x}\left(\psi_{x, t}^{*} \psi_{x, t}+\eta_{x, t}^{*} \eta_{x, t}\right)}
$$

which is true of any normal ordered function $F$ of the operators $a_{x}^{\dagger}, b_{x}^{\dagger}, a_{x}, b_{x}$.

As we indicated above, the Hamiltonian is in normal order form provided one separates the local term $e^{2} V_{x x} q_{x} q_{x}$ in the Coulomb Hamiltonian into two normal-ordered pieces

$$
e^{2} V_{x x} q_{x} q_{x}=e^{2} V_{x x}: q_{x} q_{x}:+e^{2} V_{x x}\left(a_{x}^{\dagger} a_{x}+b_{x}^{\dagger} b_{x}\right) .
$$

By reassigning the quadratic term in Eq. 4.9 to $H_{2}$, the exponent $-H \delta$ in Eq. 4.7 is normal ordered. The exponential $\exp (-H \delta)$ is not normal-ordered, but it differs from its normal ordered form by 
terms $O\left(\delta^{2}\right)$, which give a vanishing contribution to the integral in the limit of $N_{t} \rightarrow \infty$. Neglecting these terms we may replace the operator expression $\exp (-H \delta)$ with an exponential involving the fermionic fields, as follows from Eq. 4.8. We thus obtain the following expression for the partition function

$$
Z=\lim _{N_{t} \rightarrow \infty} \int \prod_{m} d \psi_{m}^{*} d \psi_{m} d \eta_{m}^{*} d \eta_{m} \times e^{-\sum_{m, n}\left(\psi_{m}^{*} M_{m, n} \psi_{n}+\eta_{m}^{*} M_{m, n} \eta_{n}\right)} e^{-\sum_{x, y, t} e^{2} Q_{x, t} V_{x, y} Q_{y, t} \delta}
$$

where $Q_{x, t}=\psi_{x, t}^{*} \psi_{x, t}-\eta_{x, t}^{*} \eta_{x, t}$ and we have used $m$ (and $n$ ) as a shorthand for the indices $x, t . M$ is a matrix whose components may be deduced from

$$
\sum_{m, n} \psi_{m}^{*} M_{m, n} \psi_{n}=\sum_{t}\left[\sum_{x} \psi_{x, t}^{*}\left(\psi_{x, t+1}-\psi_{x, t}\right)+e^{2} V_{x x} \psi_{x, t}^{*} \psi_{x, t} \delta-\kappa \sum_{\langle x, y\rangle}\left(\psi_{x, t}^{*} \psi_{y, t}+\psi_{y, t}^{*} \psi_{x, t}\right) \delta\right]
$$

where $\psi_{x, N_{t}}$ must be identified with $-\psi_{x, 0}$.

We now perform a Hubbard-Stratonovich transformation, introducing c-number real variables $\phi_{x, t}$ to recast the exponential with the quartic term in the form

$$
e^{-\sum_{x, y, t} e^{2} Q_{x, t} V_{x, y} Q_{y, t} \delta}=\int \prod_{x, t} d \phi_{x, t} e^{-\sum_{x, y, t} \phi_{x, t}\left(V^{-1}\right)_{x, y} \phi_{y, t} \delta / 4} e^{-\sum_{x, t} t e \phi_{x, t} Q_{x, t} \delta},
$$

where we have absorbed a constant measure factor in the definition of the integral over $\phi_{x, t}$. Inserting the r.h.s. of Eq. 4.12 into Eq. 4.10 and introducing the diagonal matrix $\Phi_{x, t ; y, \tau}=\left(\phi_{x, t} \delta\right) \delta_{x, y} \delta_{t, \tau}$ produces the compact result

$$
Z=\int d \phi d \psi^{*} d \psi d \eta^{*} d \eta e^{-\phi V^{-1} \phi \delta / 4-\psi^{*}(M+\imath e \Phi) \psi-\eta^{*}(M-\imath e \Phi) \eta}
$$

where we have used matrix notation for all the sums and have dropped the limit notation.

The Gaussian integration over the anticommuting variables can now be done to obtain

$$
Z=\int d \phi e^{-\phi V^{-1} \phi \delta / 4} \operatorname{det}(M-\imath e \Phi) \operatorname{det}(M+\imath \Phi \Phi)
$$

Because of the identity,

$$
\operatorname{det}(M-\imath e \Phi) \operatorname{det}(M+\imath e \Phi)=\operatorname{det}\left[(M+\imath e \Phi)^{\dagger}(M+\imath e \Phi)\right]
$$

the measure is positive definite. The down spins are treated as antiparticles (holes) moving backward in time relative to the up spins, exactly canceling the phase for each separately. Correlators for the fermion operators are now obtained by integrating the appropriate matrix elements of $(M \pm \imath \Phi \Phi)^{-1}$ with the measure given by Eq. 4.14.

Equation 4.14 is the main result of our work. It establishes the partition function and expectation values as integrals over real variables with a positive definite measure. This is a crucial step for the application of stochastic approximation methods. There remains the problem of sampling the field $\phi_{x, t}$ with a measure which contains the determinant of a large matrix. But, following what is done in lattice gauge theory, this challenge can be overcome through the application of the hybrid Monte Carlo (HMC) technique [6]. In a broad outline, in HMC one first replaces the determinants in Eq. 4.14 with a Gaussian integral over complex pseudofermionic variables $\zeta_{x, t}$ :

$$
\operatorname{det}\left[(M+\imath e \Phi)^{\dagger}(M+\imath e \Phi)\right]=\int d \zeta^{*} d \zeta e^{-\zeta^{*}(M+\imath e \Phi)^{\dagger-1}(M+\imath e \Phi)^{-1} \zeta .}
$$


(In this equation and in the following Eq. 4.16 we absorb an irrelevant, constant measure factor in the integrals.) One then introduces real "momentum variables" $\pi_{x, t}$ conjugate to $\phi_{x, t}$ and inserts in Eq. 4.15 unity written as a Gaussian integral over $\pi$. One finally arrives at

$$
Z=\int d \phi d \pi d \zeta^{*} d \zeta e^{-\phi V^{-1} \phi \delta / 4-\zeta^{*}(M+\imath e \Phi)^{\dagger-1}(M+\imath e \Phi)^{-1} \zeta-\pi^{2} / 2}
$$

The idea of HMC is to consider the simultaneous distribution of the variables $\phi, \pi, \zeta$ and $\zeta^{*}$ determined by the measure in Eq. 4.16. The phase space of these variables is explored by first extracting the $\pi, \zeta$ and $\zeta^{*}$ according to their Gaussian measure, and then evolving the $\phi$ and $\pi$ variables with fixed $\zeta, \zeta^{*}$ according to the evolution determined by the Hamiltonian

$$
\mathscr{H}(\pi, \phi)=\frac{\pi^{2}}{2}+\frac{\phi V^{-1} \phi \delta}{4}+\zeta^{*}(M+\imath e \Phi)^{\dagger-1}(M+\imath e \Phi)^{-1} \zeta .
$$

Because of Liouville's theorem, the combined motion through phase space produces an ensemble of variables distributed according to the measure in Eq. 4.16 and, in particular, of fields $\phi$ distributed according to Eq. 4.14.

Of course, the discussion above assumes that the Hamiltonian evolution of $\phi$ and $\pi$ is exact, which will not be the case with a numerical evolution. The HMC algorithm addresses this shortcoming 1) by approximating the evolution with a symplectic integrator which is reversible and preserves phase space, 2) by performing a Metropolis accept-reject step at the end of the evolution, based on the variation of the value of the Hamiltonian.

\section{Numerical tests}

We tested our method on the two-site system obtained by taking $L=1$, which can be solved exactly. We label the sites $x=0,1$. With $\kappa=1 / 3$, the Hamiltonian $H=H_{2}+H_{C}$ is now

$$
\begin{aligned}
& H_{2}=-\left(a_{1}^{\dagger} a_{0}+a_{0}^{\dagger} a_{1}+b_{1}^{\dagger} b_{0}+b_{0}^{\dagger} b_{1}\right)+\mu\left(a_{x}^{\dagger} a_{x}+b_{x}^{\dagger} b_{x}\right) \\
& H_{C}=2 e^{2}\left(a_{0}^{\dagger} a_{0}-b_{0}^{\dagger} b_{0}\right)\left(a_{1}^{\dagger} a_{1}-b_{1}^{\dagger} b_{1}\right)+\frac{2 e^{2}}{r_{0}} a_{x}^{\dagger} b_{x}^{\dagger} a_{x} b_{x}
\end{aligned}
$$

where we have taken $V_{0,1}=V_{1,0}=1 / 3$ and a local interaction term $V_{0,0}=V_{1,1}=1 / r_{0}$. The radius $r_{0}$ sets the physical scale in lattice units for localization of the net charge at the carbon atom. It must be restricted to $r_{0}<1$ for stability of the vacuum. Also the normal ordering prescription for $e^{2} V_{x x} q_{x} q_{x}$ in Eq. 4.9 adds a new contribution to $H_{2}$ in the form of an $J_{3}$ "chemical potential" $\mu a_{x, s}^{\dagger} \sigma_{3}^{s s^{\prime}} a_{x, s^{\prime}}$. It is well known [12] that a $J_{3}$ chemical potential for any value of $\mu$ does not introduce a phase in the measure. To maintain the full $S U(2)$ "flavor" symmetry of the tight-binding graphene Hamiltonian, we must set $\mu=e^{2} / r_{0}$ to its proper value. For the two-site system, the spin generators of Eq. 3.3 become

$$
J_{+}=J_{-}^{\dagger}=(-1)^{x} a_{x}^{\dagger} b_{x}^{\dagger} \text { and } J_{3}=\left[a_{x}^{\dagger} a_{x}+b_{x}^{\dagger} b_{x}\right] / 2-1,
$$

allowing us to unambiguously classify the 16 states as 5 singlets, 4 doublets and one triplet, given in Table 1. 


\begin{tabular}{|c|c|c|c|}
\hline $\mathrm{J}$ & & eigenstates $|n\rangle$ & Eigenvalues E \\
\hline $\mathrm{J}=1$ & $|0\rangle$ & $, \quad \frac{a_{0}^{\dagger} b_{0}^{\dagger}-a_{1}^{\dagger} b_{1}^{\dagger}}{\sqrt{2}}|0\rangle \quad, \quad a_{0}^{\dagger} a_{1}^{\dagger} b_{0}^{\dagger} b_{1}^{\dagger}|0\rangle$ & 0 \\
\hline $\mathrm{J}=1 / 2$ & & $\begin{array}{ccc}\frac{a_{0}^{\dagger}-a_{1}^{\dagger}}{\sqrt{2}}|0\rangle & , & \frac{a_{0}^{\dagger}-a_{1}^{\dagger}}{\sqrt{2}} b_{0}^{\dagger} b_{1}^{\dagger}|0\rangle \\
\frac{b_{0}^{\dagger}-b_{1}^{\dagger}}{\sqrt{2}}|0\rangle & , & \frac{b_{0}^{\dagger}-b_{1}^{\dagger}}{\sqrt{2}} a_{0}^{\dagger} a_{1}^{\dagger}|0\rangle \\
\frac{a_{0}^{\dagger}+a_{1}^{\dagger}}{\sqrt{2}}|0\rangle & , & \frac{a_{0}^{\dagger}+a_{1}^{\dagger}}{\sqrt{2}} b_{0}^{\dagger} b_{1}^{\dagger}|0\rangle \\
\frac{b_{0}^{\dagger}+b_{1}^{\dagger}}{\sqrt{2}}|0\rangle & , & \frac{b_{0}^{\dagger}+b_{1}^{\dagger}}{\sqrt{2}} a_{0}^{\dagger} a_{1}^{\dagger}|0\rangle \\
a_{0}^{\dagger} a_{1}^{\dagger}|0\rangle & , & b_{0}^{\dagger} b_{1}^{\dagger}|0\rangle\end{array}$ & $\begin{array}{r}3 \kappa+e^{2} / r_{0} \\
3 \kappa+e^{2} / r_{0} \\
-3 \kappa+e^{2} / r_{0} \\
-3 \kappa+e^{2} / r_{0} \\
2 e^{2}+2 e^{2} / r_{0} \\
\end{array}$ \\
\hline $\mathrm{J}=0$ & & $\begin{array}{r}\frac{\left(a_{0}^{\dagger} b_{0}^{\dagger}+a_{1}^{\dagger} b_{1}^{\dagger}\right) \cos \theta-\left(a_{0}^{\dagger} b_{1}^{\dagger}+a_{1}^{\dagger} b_{0}^{\dagger}\right) \sin \theta}{\sqrt{2}}|0\rangle \\
\frac{\left(a_{0}^{\dagger} b_{0}^{\dagger}+a_{1}^{\dagger} b_{1}^{\dagger}\right) \sin \theta+\left(a_{0}^{\dagger} b_{1}^{\dagger}+a_{1}^{\dagger} b_{0}^{\dagger}\right) \cos \theta}{\sqrt{2}}|0\rangle \\
\frac{a_{0}^{\dagger} b_{1}^{\dagger}-a_{1}^{\dagger} b_{0}^{\dagger}}{\sqrt{2}}|0\rangle\end{array}$ & $\begin{array}{r}\sqrt{36 \kappa^{2}+\left(1-1 / r_{0}\right)^{2} e^{4}}-e^{2}+e^{2} / r_{0} \\
-\sqrt{36 \kappa^{2}+\left(1-1 / r_{0}\right)^{2} e^{4}}-e^{2}+e^{2} / r_{0} \\
-2 e^{2}+2 e^{2} / r_{0}\end{array}$ \\
\hline
\end{tabular}

Table 1: Spin multiplets and energies for the 2 site Graphene model.

We compared HMC results for expectation values of several products of fermionic operators with the corresponding exact values, finding satisfactory agreement. For example, the correlation function

$$
C_{a}(t)=\left\langle\left(a_{0}-a_{1}\right)(t)\left(a_{0}^{\dagger}-a_{1}^{\dagger}\right)(0)\right\rangle / 2
$$

is illustrated in Figure. 5, which shows HMC results converging to the exact correlators for both the free theory with $e=0$ and an interacting case with $e=0.5$.

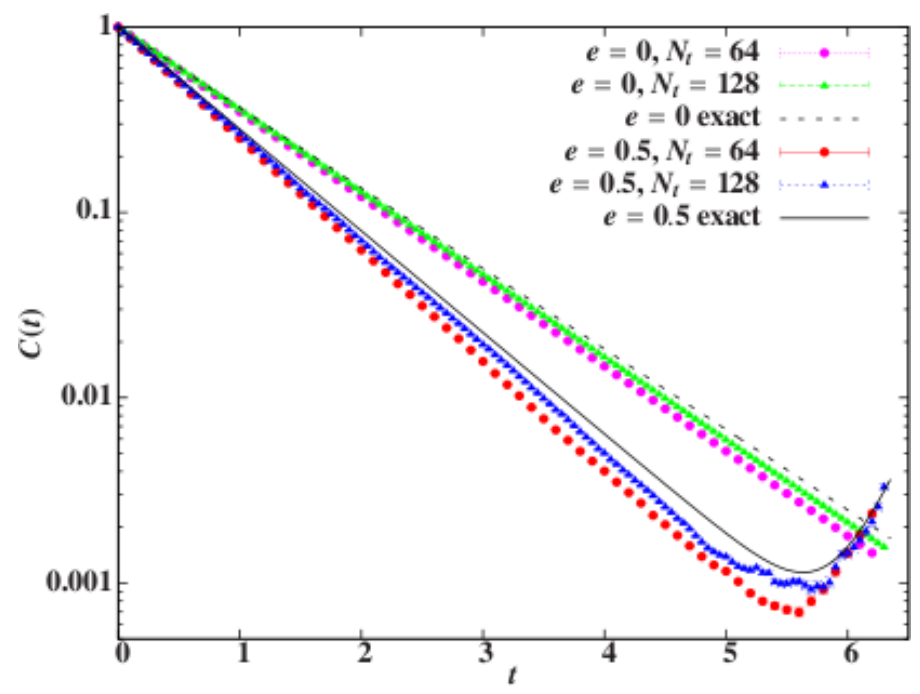

Figure 5: HMC results for $C_{a}(t)$ (Eq. 5.2) with $e=0$ and $e=0.5$ compared to the exact correlators. Here $\mu=e^{2} / r_{0}, r_{0}=1 / 2$ and $\beta=N_{t} \delta=6.4$.

A stringent test is to demonstrate the convergence to exact $S U(2)$ symmetry in the "time" continuum limit. To this end, consider a second correlation function,

$$
C_{b}(t)=\left\langle\left(b_{0}^{\dagger}+b_{1}^{\dagger}\right)(t)\left(b_{0}+b_{1}\right)(0)\right\rangle / 2,
$$




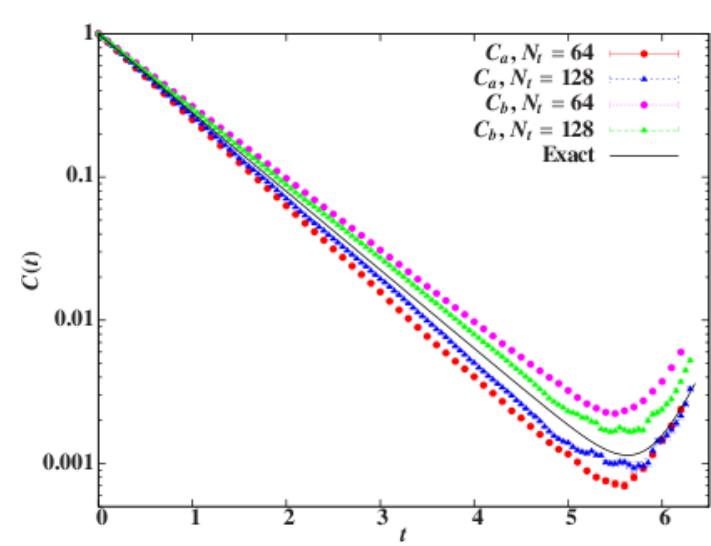

Figure 6: HMC results for $C_{a}(t)$ (Eq. 5.2) and $C_{b}(t)$ (Eq. 5.3) with $e=0.5$ compared to the exact correlator. As in Figure. 5, $\mu=e^{2} / r_{0}, r_{0}=1 / 2$ and $\beta=N_{t} \delta=6.4$.

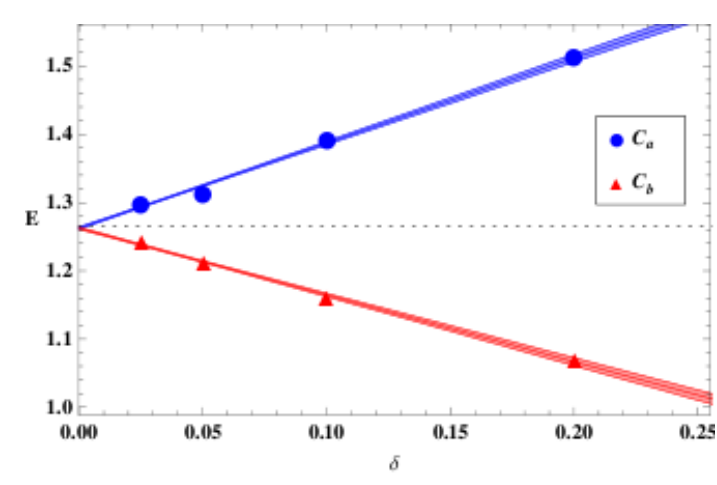

Figure 7: Linear extrapolation (with error band) of the HMC energies for doublet correlators $C_{a}$ and $C_{b}$ as a function of the "time" lattice spacing $\delta=$ $6.4 / N_{t}$ for $N_{t}=32,64,128$ and 256. The dotted horizontal line marks $E_{0}=1.266$.

related to $C_{a}(t)$ by an $S U(2)$ rotation. Figure. 6 illustrates that HMC results for both $C_{a}(t)$ and $C_{b}(t)$ converge to the same continuum limit.

We extract the energies of the doublet states at nonzero $\delta=\beta / N_{t}$ by fitting the correlator data in Figure 6 to single exponentials, $C(t) \approx e^{-E t}$ for fit range $0.4<t<4$. The results in Figure. 7 clearly show linear behavior $E \approx E_{0}+c_{1} \delta$, converging to the exact continuum $E_{0}=e^{2}+$ $\sqrt{4+e^{4}}-1 \approx 1.266$. The continuum limit is consistent with restoration of the $S U(2)$ symmetry of the Hamiltonian: a joint linear fit to both sets of energies gives $\lim _{\delta \rightarrow 0} E=1.262 \pm 0.004$, with $c_{1}=1.25 \pm 0.07$ and $-0.98 \pm 0.04$ for correlators $C_{a}$ and $C_{b}$, respectively.

\section{Discussion}

Before scaling up our simulations to large lattices, it is useful to reflect on the formalism presented here. In our formulation, the theory is reduced to a $2+1$ dimensional lattice theory with no sign problem. The non-local potential when expressed as a convolution can be computed efficiently using a Fast Fourier transform. The only approximation to the tight binding Hamiltonian for the nearest neighbor coupling is the discretization of the Euclidean time, $\delta$. So as we extrapolate to $\delta \rightarrow 0$, we can in principle solve the exact tight binding model numerically to arbitrary precession. However there are, as in all lattice field theories, improved discretization and algorithms that may accelerate the convergence. The difference here is the "continuum limit" involves only the "time" discretization and there is no symmetry rotating this axis into the hexagonal plane. So we are exploring alternative discretization in "time" which may improve computational efficiency and accuracy for small $\delta$.

On a theoretical level, it is instructive to re-derive the discretization for finite lattice spacing $\delta$ starting with our exact time continuum $(\delta=0)$ acton or its Lagrangian $\left(S=\int d t \mathscr{L}\right)$ derived above,

$$
\mathscr{L}(t)=\psi_{x}^{\dagger}(t)\left(\partial_{t}+i e \sigma_{3} \phi_{x}(t)\right) \psi_{x}(t)+e^{2} V_{x x} \psi_{x}^{\dagger}(t) \psi_{x}(t)-\kappa \sum_{\langle x, y\rangle} \psi_{x}^{\dagger}(t) \psi_{y}(t)+\frac{1}{4} \phi_{x}(t) V_{x y}^{-1} \phi_{y}(t),
$$


where for simplicity we have combined the two spin components $\psi, \eta$ into a spinor field: $\psi_{x}^{\dagger}=$ $\left(\psi_{x}^{*}, \eta_{x}^{*}\right)$ and $\psi_{x}=\left(\psi_{x}, \eta_{x}\right)^{T}$. Let us also introduce a a staggered mass parameter, $+/-m \psi_{x}^{\dagger} \psi_{x}$ for sites on the $\mathrm{A} / \mathrm{B}$ lattices respectively, which will be useful in numerical simulation in any case due to critical slowing down in the chiral limit. The result for the free theory $(e=0)$ on the nearest neighbor hexagonal lattice is to lift the zero mode symmetrically to give the continuum dispersion relation,

$$
E= \pm i \sqrt{\omega_{k}^{2}+m^{2}}
$$

At $m=0$ this is just the well know spectrum of the nearest neighbor graphene Hamiltonian $H_{2}$ by going to Fourier space. Using the oblique reciprocal co-ordinates, $k_{1}=3 k_{x} / 2+\sqrt{3} k_{y} / 2, k_{2}=\sqrt{3} k_{y}$, on the Hexagonal lattice described in the caption of Figure.6, $\omega_{k}$ is given by $\omega_{k}=\left|1+e^{i k_{1}}+e^{i k_{2}}\right|$ in spatial units set by taking $\kappa=1$. On a finite periodic lattice, the momenta are discrete $\left(k_{i}=2 \pi n_{i} / L_{i}\right.$ for integer $n_{i}$ ), so to preserve the exact zero modes at the apex of the Dirac cones, $k_{1}=-k_{2}=$ $\pm 2 \pi / 3$, we must chose $L_{i}$ to be divisible by 3 .

Now let us re-introducing the temporal lattice spacing, $\delta$, into our continuum Lagrangian (6.1). Our derivation above amounted to replacing the derivative by a forward difference, $\partial_{t} \psi_{x} \rightarrow$ $\left(\psi_{x, t+1}-\psi_{x, t}\right) / \delta$, but there are other equally valid discretizations with the same continuum limit. Another attractive choice is to use this forward difference on the A lattice, $\partial_{t} \psi_{x} \rightarrow\left(\psi_{x, t+1}-\psi_{x, t}\right) / \delta$, but a backward difference on the B lattice, $\partial_{t} \psi_{x} \rightarrow\left(\psi_{x, t}-\psi_{x, t-1}\right) / \delta$, which has an interesting lattice symmetry of time reversal times $\mathrm{A} / \mathrm{B}$ lattice exchange. Comparing the free $(e=0)$ dispersion relation at finite $\delta$, we have

$$
(2 / \delta) e^{i E \delta / 2} \sin (E \delta / 2)= \pm i \sqrt{\omega_{k}^{2}+m^{2}}
$$

for our original choice of all forward differences versus

$$
(2 / \delta) \sin (E \delta / 2)= \pm i \sqrt{\frac{\omega_{k}^{2}+m^{2}}{1-m \delta}} .
$$

for the A/B alternating forward/ backward difference choice. Both dispersion relations of course yield the well know exact continuum dispersion relation (6.2) for $\delta \rightarrow 0$. However, the second one is accurate to $O\left(\delta^{2}\right)$ and has a symmetric spectrum in the complex plane characteristic of a relativistic fermion in the continuum. Very likely this form has some real advantages which we are actively investigating.

Next let us return to the interacting case. Note in our Lagrangian (6.1), that the quadratic (kinetic) action for the electrons exhibits a 1-d space independent "gauge invariance"

$$
\psi_{x}(t) \rightarrow \exp \left[-i e \sigma_{3} \theta(t)\right] \psi_{x}(t), \quad \psi_{x}^{\dagger}(t) \rightarrow \psi_{x}^{\dagger}(t) \exp \left[i e \sigma_{3} \theta(t)\right], \quad \phi_{x}(t) \rightarrow \phi_{x}(t)+\partial_{t} \theta(t),
$$

much like the residual gauge invariance of the 4 -d representation with gauge potential $A_{0}$. However $\phi$ is not the same object. The potential term in $\mathscr{L}$ is not gauge invariant and thus it fixes this residual gauge transformation. It is natural to ask what happens if we preserve this gauge invariance for the kinetic term on the temporal lattice. We find an intriguing connection between the normal ordering term, $e^{2} V_{x x} \psi_{x}^{\dagger} \psi_{x}$, and the gauge invariance of the kinetic term, which suggest another discretization using compact gauge variables on the links,

$$
\int d t \psi_{x}^{\dagger}(t)\left(\partial_{t}+i e \phi_{x}(t) \sigma_{3}\right) \psi_{x}(t) \rightarrow \sum_{t} \psi_{x, t}^{\dagger}\left(e^{i e \delta \sigma_{3} \phi_{x, t}} \psi_{x, t+1}-\psi_{x, t}\right)
$$


which like Wilson link variables in lattice field theory preserve gauge invariance of the kinetic term at the discrete times $t$ for each temporal slice. Now let us expand in $\delta$ as usual to see how this approaches the continuum Lagrangian (6.1):

$$
\sum_{t} \psi_{x, t}^{\dagger}\left(e^{i e \delta \sigma_{3} \phi_{x, t}} \psi_{x, t+1}-\psi_{x, t}\right) \simeq \int d t \psi^{\dagger} \partial_{t} \psi_{x}+i e \int d t \psi_{x}^{\dagger} \sigma_{3} \phi_{x} \psi_{x}-\delta \int d t \frac{e^{2}}{2} \phi_{x}^{2} \psi_{x}^{\dagger} \psi_{x}+\cdots
$$

The first and two second term on the right hand side are the required form to account for the Hubbard-Stratonovich transformation. Usually we would drop the third term as irrelevant in the continuum. However the Hubbard-Stratonovich field, $\phi_{x, t}$, is not a dynamical field and on the lattice its fluctuations are controlled only by the quadratic potential in the action,

$$
\frac{1}{4} \int d t \phi_{x}(t) V_{x y}^{-1} \phi_{y}(t) \rightarrow \sum_{t} \frac{\delta}{4} \phi_{x, t} V_{x y}^{-1} \phi_{y, t}
$$

This term implies that the variance for small $\delta$ diverges on each time slice: $\left\langle\phi^{2}\right\rangle=O(1 / \delta)$. Consequently the third term is not suppressed and must be taken into account. Remarkably it can be shown that this term is exactly equivalent to adding the normal ordering term to leading order in $\delta$. To show this we make a field redefinition: $\phi_{x} \simeq \widetilde{\phi}_{x}-\delta V_{x y} \widetilde{\phi}_{y} \psi_{y}^{\dagger} \psi_{y}$ which when substituted into the lattice Lagrangian cancels the $\phi_{x}^{2}$ term in Eq. 6.7 to leading order but to preserve the measure in the path integral also requires us to introduce the Jacobian of this change of variables. This Jacobian gives rise to our normal ordering term in the continuum limit,

$$
\int \mathscr{D} \phi_{x, t}=\int \mathscr{D} \widetilde{\phi}_{x, t} \operatorname{det}\left[\frac{\partial \phi_{x, t}}{\partial \widetilde{\phi}_{x^{\prime}, t^{\prime}}}\right]=\int \mathscr{D} \widetilde{\phi}_{x, t} e^{\operatorname{Tr} \log (1-\delta V \psi \psi)} \simeq \int \mathscr{D} \widetilde{\phi}_{x, t} e^{-\int d t V_{x x} \psi_{x}^{\dagger}(t) \psi_{x}(t)}
$$

In summary an alternative to the normal ordering term on the lattice is to ignore it and introduce compact gauge links.

An independent derivation that yields the same result is to reverse the argument in Sec. 4 by first introducing the Hubbard-Stratonovich transformation in the Hamiltonian for each of the $N_{t}=\beta / \delta$ factors in the discretized transfer matrix,

$$
e^{-\beta H}=e^{-H \delta} e^{-H \delta} \ldots e^{-H \delta} \quad\left(N_{t} \text { terms }\right) .
$$

Since the local charges commute $\left[q_{x}, q_{y}\right]=0$ the result of the Hubbard-Stratonovich transformation to leading order is simply the replacement,

$$
e^{-H \delta}=\int \Pi_{x} d \phi_{x, t} e^{-H_{2} \delta-i e \delta \phi_{x, t} q_{x}+\frac{\delta}{4} \phi_{x, t} V_{x y}^{-1} \phi_{y, t}}
$$

for each factor on time slice $t$. Now if we use the exact normal ordering identity,

$$
\exp \left[-i e \delta \phi_{x} a_{x}^{\dagger} \sigma_{3} a_{x}\right] \rightarrow \exp \left[\psi_{x}^{\dagger} e^{-i e \delta \sigma_{3} \phi_{x}} \psi_{x}\right]
$$

given as Eq. A8 in Ref. [13] and expand to leading order, we again get the quadratic (seagull) term, $-\left(e^{2} / 2\right) \delta^{2} \phi_{x}^{2} \psi_{x}^{\dagger} \psi_{x}$, in Eq. 6.7 . To formally take the continuum limit with this term of course we must again make a change of variables arriving to the unique continuum Lagrangian (6.1). 
This alternate derivation makes it clear that the two lattice expressions (with $-\left(e^{2} / 2\right) \delta^{2} \phi_{x}^{2} \psi_{x}^{\dagger} \psi_{x}$ or $e^{2} V_{x x} \psi_{x} \psi_{x}$ ) are simply different approximation to account for normal ordering. What is surprising is that compact link variables also provide this contribution and that in this form there is no explicit dependence on $V_{x x}$ need to define the effects of normal ordering. The dependence is implicit in the necessity to stabilize the quadratic form with a local repulsion at the Carbon ions to allow the Hubbard-Stratonovich transformation to be legitimate.

Beyond the curiosity of this seagull term inducing the normal ordering correction, it opens up the attractive option of using compact links, as if $\phi$ were a gauge potential. One might suppose that this is a consequence of dimensional reduction from a real 4-d gauge theory but it is also more general since the result is exact for any static potential term $V_{x y}$. The generality of the construction allows any static potential and any fixed geometry for the graphene sheet. It is also possible to include fluctuation of the graphene sheet by modeling phonon interactions with additional dynamical spatial gauge links. At present we are investigating a range of discretization schemes numerically in comparison with solving the exact spectrum on lattices with $L_{1}=L_{2}=2$ to see how each converges to the continuum limit. These test lattices have 8 sites with two spins per site so in total $2^{16}=65536$ states offer a rigorous and non-trivial verification of our HMC algorithm and software.

\section{Conclusion}

We have presented a Lagrangian formalism for the nearest neighbor tight binding theory for single layer graphene that allows one to do Hybrid Monte Carlos simulations by the traditional method of Euclidean lattice field theory.

While the results we reported are for a small single hexagonal cell test systems, they demonstrate that HMC simulations of graphene directly on the hexagonal graphene lattice are possible and have the potential to produce valuable results. The dominant nearest neighbor hopping term has no sign problem, and we anticipate that a small next-to-nearest neighbor coupling $\kappa^{\prime} / \kappa \simeq 0.03$ can be accommodated by reweighting without a prohibitive cost. The crucial observation is the cancellation between the phase of the up spin and down spin determinant, when the latter are treated as holes moving backward in time. The only approximation is the discretization error introduce by a lattices spacing $\delta$ for the "time" or temperature lattice. This opens up a method for effectively solving this tight binding model numerically to arbitrary precision subject to taking the 1-d continuum limit. Relative to computational cost of lattice QCD simulations, clearly lattice graphene simulations are not only feasible but considerably more tractable with modern computing resources and algorithms. Still this is a young computational field and there is much work to be done to explore both algorithms and discretization scheme to optimize these simulations.

We are currently pursuing simulations of larger systems, and beginning to explore the many possible generalizations such as distortions of the lattice, phonons, inclusion of magnetic fields, etc. We have a fully functioning code for our periodic hexagonal lattice with nearest neighbor hopping terms and a periodic Coulomb potential. We are putting the conjugate gradient inverter and the molecular dynamics evolution onto single GPUs. Since the NVIDIA Tesla class GPU have device memories in the range of 3 to 6 GigaBytes, this will allow a very substantial on card lattice volumes and a performance of $\mathrm{O}(100)$ Gigaflops per GPU. The potential problems this open up to simulation are substantial. Graphene has a range of interesting properties. Our first goal is to repeat the 
calculation in Ref. $[4,5]$ of the critical charge for the excitation gap (or chiral symmetry breaking) for single layer graphene due to the strong Coulomb potential. Having a reliable number for this critical parameter for the tight binding model is of considerable experimental interest. Beyond that there are a large range of effects due to changing boundary conditions and the introduction of phonons, which are easily introduced into the lattice Lagrangian formalism presented here. It is even feasible to simulate small graphene samples with lattices, identical in size and geometry to those being studied in experimental investigation. Here finite size effects are real and very interesting, both theoretically and technologically.

Acknowledgments: We wish to acknowledge the many fruitful conversations with Dr. Ronald Babich, Prof. Antonio Castro Neto, Prof. Claudio Chamon and Dr. MIchael Cheng during the course of this research and support under DOE grants DE-FG02-91ER40676, DE-FC02-06ER41440, and NSF grants OCI-0749317, OCI-0749202. Part of this work was completed while two of the authors were at the Aspen Center for Physics.

\section{References}

[1] K. S. Novoselov et al., "Electric Field Effect in Atomically Thin Carbon Films", Science 306, 666 (2004).

[2] A. H. Castro Neto et al., "The electronic properties of graphene", Rev. Mod. Phys. 81, 109 (2009).

[3] H. J. Rothe, Lattice Gauge Theories: An Introduction, (World Scientific, Singapore, 2005).

[4] J. E. Drut and T. A. Lähde, "Is Graphene in Vacuum an Insulator?", Phys. Rev. Lett. 102, 026802 (2009); Phys. Rev. B 79, 165425 (2009).

[5] T. A. Lahde and J. E. Drut, "Strongly Coupled Graphene on the Lattice," arXiv:1111.0929 [hep-lat].

[6] Simon Duane et al., "Hybrid Monte Carlo" Phys. Lett. B 195, 216 (1987).

[7] J. Giedt, A. Skinner and S. Nayak, "Effects of flavor-symmetry violation from staggered fermion lattice simulations of graphene," arXiv:0911.4316 [cond-mat.str-el].

[8] T.O. Wehling, E. Sastioglu, C> Friedrich, A > I. Lichtenstein, M. I. Katsnelson andS. Blugel, "Strength of Effective Coulomb Interactions in Graphene and Graphite" Phys. Rev. Lett. 106, 236805 (2011).

[9] J. W. Negele and H. Orland, Quantum Many Particle Systems, (Addison-Wesley, Redwood City, California, 1988).

[10] R. L. Stratonovich, "On a Method of Calculating Quantum Distribution Functions", Sov. Phys. Dokl. 2, 416 (1958); J. Hubbard, "Calculation of Partition Functions", Phys. Rev. Lett. 3, 77 (1959).

[11] R. Blankenbecler, D. J. Scalapino and R. L. Sugar, "Monte Carlo calculations of coupled boson-fermion systems. I" Phys. Rev. D 24, 2278 (1981).

[12] M. G. Alford, A. Kapustin and F. Wilczek, "Imaginary chemical potential and finite fermion density on the lattice", Phys. Rev. D 59, 054502 (1999); D. T. Son and M. A. Stephanov, "QCD at finite isospin density", Phys. Rev. Lett. 86, 592 (2001).

[13] M. Luscher, "Construction Of A Selfadjoint, Strictly Positive Transfer Matrix For Euclidean Lattice Gauge Theories," Commun. Math. Phys. 54, 283 (1977). 